\section{Regards sur l'économie allemande}

Bulletin économique du CIRAC

$71 \mid 2005$

Varia

\title{
Salaire minimum : le cas particulier du BTP
}

Isabelle Bourgeois

\section{OpenEdition}

Journals

Édition électronique

URL : https://journals.openedition.org/rea/342

DOI : $10.4000 /$ rea. 342

ISBN : 978-2-8218-0838-6

ISSN : 1965-0787

Éditeur

CIRAC

Édition imprimée

Date de publication : 1 mai 2005

Pagination : 31-32

ISSN : 1156-8992

Référence électronique

Isabelle Bourgeois, "Salaire minimum : le cas particulier du BTP », Regards sur l'économie allemande [En ligne], 71 I mai 2005, document 2, mis en ligne le 23 juin 2008, consulté le 28 juin 2022. URL : http://journals.openedition.org/rea/342 ; DOI : https://doi.org/10.4000/rea.342

Ce document a été généré automatiquement le 29 septembre 2020

(C) CIRAC 


\title{
Salaire minimum : le cas particulier du BTP
}

\author{
Isabelle Bourgeois
}

\section{La Loi $A E n t G$ de 1996 introduit le principe du pays d'accueil}

1 La loi du 26-02-1996, surnommée Arbeitnehmer-Entsendegesetz (AEntG) et entrée en vigueur le $1^{\text {er }}$ mars de la même année, introduit pour l'essentiel des salaires minima dans le secteur du BTP. Elle transpose en droit national, avant même son adoption définitive, la « Directive 71/96/CE du parlement européen et du conseil du 16 décembre 1996 concernant le détachement des travailleurs effectué dans le cadre d'une prestation de services ». La loi AEntG introduit clairement le principe du pays d'accueil à ce secteur en lui appliquant les dispositions contractuelles de diverses conventions conclues dans le BTP et qui avaient obtenu valeur de loi en vertu de l'article de la Loi sur les conventions tarifaires de branche (Tarifvertragsgesetz) permettant la " déclaration d'utilité générale " (Allgemeinverbindlichkeit) des accords contractuels de branche sous certaines conditions. Le nom complet de la loi de 1996 est parlant à cet égard: "Loi sur les conditions de travail contraignantes pour les services transfrontaliers » (Gesetz über zwingende Arbeitsbedingungen bei grenzüberschreitenden Dienstleistungen). Elle s'applique à quatre catégories d'activités du BTP.

\section{Elle crée des salaires minima pour 700000 salariés}

2 Ces minima concernent les quelque 700000 salariés du BTP, qu'ils soient allemands ou ressortissants d'un autre Etat membre. Par rapport aux salaires moyens versés dans les quatre types de métiers couverts par la loi, ces minima en représentent entre $42 \%$ (pour un OS plâtrier-peintre dans les nouveaux Länder) et $73 \%$ (pour un salarié embauché à l'ouest dans le secteur «activité principale du BTP »). Le contrôle du respect des dispositions de la loi $A E n t G$ incombe à l'administration des douanes. Depuis 
un amendement à la loi (§ 3) entré en vigueur le $1^{\mathrm{er}}$ janvier 2004, les entreprises de BTP ayant leur siège hors du territoire allemand et souhaitant offrir des prestations en RFA sont soumises à un régime de déclaration renforcé : chaque chantier doit être déclaré avant ouverture auprès de la Direction Contrôle financier du travail illégal de la direction régionale des Finances de Cologne.

Mimima légaux en vigueur dans le BTP à la fin 2004 selon la Loi AEntG (salaires bruts)

\begin{tabular}{|l|l|c|c|c|c|}
\hline & & \multicolumn{2}{|c|}{ En € par heure } & \multicolumn{2}{c|}{ En € par mois*) } \\
\hline & salariés & formés sur le tas & qualifiés & formés sur le tas & qualifiés \\
\hline Activité principale du BTP & ouest & 10,36 & 12,47 & 1758 & 2116 \\
\hline (520 000 salariés) & est & 8,95 & 10,01 & 1518 & 1690 \\
\hline Démontage/démolition & ouest & 9,49 & 11,60 & 1527 & 1867 \\
\hline (11 000 salariés) & est & 8,95 & 9,65 & 1557 & 1679 \\
\hline Peinture/revêtements & ouest & 7,69 & 10,53 & 1338 & 1832 \\
\hline (180 000 salariés) & est & 7,00 & 9,20 & 1218 & 1601 \\
\hline Travaux de charpente $\left.{ }^{*}\right)$ & ouest & 9,30 & 9,30 & 1578 & 1578 \\
\hline (84 000 salariés) & est & 9,30 & 9,30 & 1578 & 1578 \\
\hline
\end{tabular}

Sources : Ministère Fédéral de l'Economie et du Travail, Destatis, iwd 15/05, Handelsblatt, 8/10-04-05) *) Calculés sur la base de la durée hebdomadaire conventionnelle, soit 39 heures, sauf dans la peinture (40 h à l'est et $37 \mathrm{~h}$ à l'ouest). **) Salaires unifomisés dans ces métiers.

\section{C'est une mesure de lutte contre le travail illégal}

3 L'objectif déclaré de cette loi est en effet de lutter contre le travail au noir. Le site des Douanes allemandes (www.zoll.de), où est expliquée la réglementation et où on peut effectuer les démarches administratives nécessaires, l'expose très clairement. Voici la présentation qui figure sous le titre "Détachement de travailleurs " (Entsendung von Arbeitnehmern) : "De plus en plus d'entreprises étrangères, principalement en provenance des autres Etats membres de l'UE, font leur entrée massive dans le secteur du BTP allemand pour y proposer leurs services à des prix défiant toute concurrence [Billigstangebote]. Dans l'intérêt d'une concurrence loyale et de la préservation des intérêts des salariés est entrée en vigueur le $1^{\text {er }}$ mars 1996 la Loi sur les conditions de travail contraignantes pour les services transfrontaliers : la Loi AEntG ». 


\section{Ces minima légaux sont très élevés en comparaison des minima de branche}

$4 \quad$ L'objectif quelque peu protectionniste des salaires minima fixés par cette loi (qui sont nettement supérieurs au SMIC moyen français, soit $1185 €$ par mois selon Eurostat) est donc très différent de celui des salaires planchers fixés par les partenaires sociaux dans le cadre normal de la convention collective de branche. La comparaison avec le montant des minima conventionnels de branche abonde dans ce sens. Dans le secteur bancaire, par exemple, il est actuellement de 10,30€ par heure (il passera à 10,50€ en septembre) pour les ouvriers comme les employés; c'est l'un des plus élevés. Dans l'industrie métallurgique de Rhénanie du Nord-Westphalie, il n'est que de 9,35€ pour un ouvrier et de 7,38€ pour un employé - c'est moins que le salaire horaire minimum d'un plâtrier-peintre ouest-allemand tel que défini par la loi AEntG.

Les minima conventionnels de branche les plus bas d'Allemagne (en $€ /$ heure)

\begin{tabular}{|l|c|c|l|c|c|}
\hline & ouest & est & & ouest & est \\
\hline \hline Pâtisserie industrielle & 6,74 & 8,91 & Horticulture/cult. maraîchère & 5,24 & 2,75 \\
\hline Commerce de détail & 6,49 & 6,71 & Hôtellerie/gastronomie & 5,18 & 4,61 \\
\hline Boucherie & 6,21 & 5,49 & Sécurité & 5,10 & 4,15 \\
\hline \hline Transports & 6,10 & 3,91 & Agriculture & 4,68 & 4,19 \\
\hline Entretien BTP & 6,05 & 6,36 & Coiffure & 3,38 & 3,05 \\
\hline
\end{tabular}

Source : Die Zeit, 14-04-05. NB : on trouve la liste détaillée de tous les minima de branche sur le site de la Fondation Hans Böckler, l'institut de recherche proche du DGB, qui recense et actualise régulièrement tous les salaires conventionnels allemands : www.wsi.de.

5 Au vu de ces montants, on comprend mieux certains des enjeux du débat entretenu outre-Rhin à propos du projet de directive 'Services' (voir dans ce numéro). Généraliser la pratique du salaire minimum légal, ainsi que le plaident de nombreux acteurs, le syndicat des services ver.di en tête, signifie dès lors nécessairement revendiquer une hausse des salaires dans la plupart des secteurs. Car selon les études de l'institut WSI (citées par Die Zeit, 14-04-05), quelque 3,4 millions de salariés allemands gagnaient moins de $8,75 €$ par heure en 2003. On peut douter que ce choix soit de nature à résoudre les problèmes structurels du marché de l'emploi allemand.

\section{INDEX}

Mots-clés : bâtiment, BTP, industrie, salaire minimum 